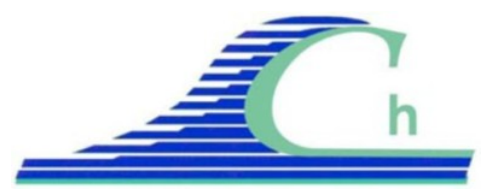

XII ${ }^{\text {ìmes }}$ Journées Nationales Génie Côtier - Génie Civil

Cherbourg, 12-14 juin 2012

DOI:10.5150/jngcgc.2012.116-M C Editions Paralia CFL

disponible en ligne - http://www.paralia.fr - available online

\title{
Aptitude au traitement et comportement mécanique des sédiments de dragage
}

\author{
Walid MAHERZI $^{1,2}$, Lucile SAUSSAYE ${ }^{1,2}$, Mohamed BOUTOUIL ${ }^{1,2}$
}

1. ESITC Caen, F-14610 Epron, France.

2. Université de Caen Basse-Normandie (UCBN), Unité de Recherche Aliments Bioprocédés Toxicologie Environnements (UR ABTE) EA 4651, Bd Maréchal Juin, 14032 Caen, France.

doctorant.wm@esitc-caen.fr ; ecr.geotech@esitc-caen.fr ;

dir.recherche@esitc-caen.fr

\section{Résumé :}

Les résultats présentés dans cet article s'inscrivent dans le cadre du projet de recherche Européen SETARMS dont l'un des objectifs est de mettre en place des solutions innovantes et durables de gestion des sédiments marins dragués en Manche (FranceAngleterre) pour une application en techniques routières.

Après la détermination des caractéristiques physiques et géotechniques de 8 sédiments français, phase à l'issue de laquelle leur est attribuée une classe GTR (LCPC-SETRA, 2000), les recommandations du GTS (LCPC-SETRA, 2000) ont été utilisées pour le traitement des sédiments aux liants hydrauliques.

A la suite de l'application d'une formulation de traitement avec 3\% de chaux vive et $6 \%$ de ciment CEMII/B 32,5R, une phase de caractérisation physique et mécanique est menée. Les critères à atteindre vise une réutilisation du matériau traité en couche de forme. Des essais d'aptitude au traitement comprenant des mesures de gonflements volumiques $(\mathrm{Gv})$ et de résistances à la traction indirecte $\left(\mathrm{R}_{\mathrm{it}}\right)$ ont été réalisés. Ils sont complétés par des essais de résistance à la compression simple $\left(\mathrm{R}_{\mathrm{c}}\right)$ et de $\mathrm{R}_{\mathrm{it}}$ en cure normale.

Les résultats obtenus montrent que malgré des classes GTR (LCPC-SETRA, 2000) assez proches (F11 et F12), les sédiments répondent différemment aux essais d'aptitude. Les quatre sédiments répondant le plus favorablement à ce simple traitement aux liants hydrauliques sont repris afin de développer de nouvelles formulations, plus prometteuses.

\section{Mots-clés :}

Sédiments de dragage marins - Essai d'aptitude - Liants hydrauliques - Valorisation Techniques routières 


\section{Introduction}

Le dragage est une opération fondamentale pour l'exploitation des infrastructures portuaires. Les volumes dragués annuellement dans le monde représente plus de $700 \mathrm{Mm}^{3}$ tandis qu'en France leur volume est évalué à $50 \mathrm{Mm}^{3}$ (BOUTOUIL, 2010). Avec une législation de plus en plus sévère visant à préserver l'environnement et les milieux aquatiques et interdisant le clapage en mer de sédiments contaminés, la gestion des sédiments de dragage à terre est devenue une nécessité.

La mise en place du Grenelle de l'Environnement et du Grenelle de la Mer amène à reconsidérer cette ressource face aux besoins en matériaux granulaires, estimés à $400 \mathrm{Mt}$ par an dans le seul secteur du génie civil.

Le projet franco-britannique SETARMS prend en considération ces problématiques et les transposent à l'espace Manche. Les sédiments dont il sera question dans cet article ont été prélevés au cours des années 2010 et 2011 dans 8 ports de la façade française de la Manche, les prélèvements ont été effectués dans les bassins principaux des ports étudiés. Ils ont fait l'objet d'une étude géochimique, réalisée par le Laboratoire d'Analyses, de Surveillance et d'Expertise de la Marine (LASEM) de Cherbourg et l'Unité de Recherche Aliments Bioprocédés Toxicologie et Environnement (ABTE) de l'Université de Caen Basse-Normandie (UCBN), partenaires du projet. Afin de garantir la confidentialité des données aux ports partenaires, les sédiments étudiés sont codés.

\section{Matériaux et méthodes}

\subsection{Classification géotechnique des sédiments étudiés}

Une caractérisation physique et mécanique des sédiments de dragage selon le Guide technique des Terrassements Routiers - Réalisation des remblais et des couches de forme (GTR) (LCPC-SETRA, 2000) a été menée.

Le tableau 1 résume les principales caractéristiques des 8 sédiments. Les sédiments A001, B001, D001, E001 et G001 sont classés comme des matériaux de type F11, alors que les sédiments C001, F001 et M001 sont classés comme des matériaux de classe F12. Au-delà de la prise en compte de la teneur en matière organique, les caractéristiques physiques des sédiments révèlent un comportement similaire aux matériaux des classes A1 ou A2.

Selon le GTR (LCPC-SETRA, 2000), les matériaux de classes A1 et A2, à faibles teneurs en M.O. (M.O. < 3\%) sont considérés comme de bons matériaux en terrassement et peuvent être traités aux liants hydrauliques pour une utilisation en souscouches routières. Dans le cas contraire (M.O. $\geq 3 \%$ ), les matériaux doivent faire l'objet d'une étude spécifique afin d'évaluer leurs comportements vis-à-vis d'un traitement aux liants hydrauliques. 


\section{XII ${ }^{\text {èmes }}$ Journées Nationales Génie Côtier - Génie Civil \\ Cherbourg, 12-14 juin 2012}

Tableau 1. Classification GTR (LCPC-SETRA, 2000) des sédiments de dragage marins.

\begin{tabular}{|c|c|c|c|c|c|c|c|c|}
\hline Echantillons & $A 001$ & $B 001$ & $\mathrm{C} 001$ & $D 001$ & $E 001$ & $F 001$ & G001 & M001 \\
\hline M.O. (\%) & 10.3 & 4.8 & 14.1 & 7.6 & 5.4 & 11.4 & 10.7 & 17.1 \\
\hline $\begin{array}{l}\text { F. Argileuse } \\
0 / 2 \mu m(\%)\end{array}$ & 7.3 & 20.2 & 10.0 & 10.0 & 14.0 & 4.0 & 19.6 & 2.0 \\
\hline $\begin{array}{l}\text { F. silteuse } \\
2 / 63 \mu m(\%)\end{array}$ & 38.0 & 67.4 & 35.7 & 40.5 & 33.5 & 24.0 & 31.4 & 58.0 \\
\hline $\begin{array}{c}F . \text { sableuse } \\
63 \mu \mathrm{m} / 2 \mathrm{~mm}(\%)\end{array}$ & 54.7 & 12.4 & 54.3 & 49.5 & 52.5 & 71.0 & 49.0 & 40.0 \\
\hline 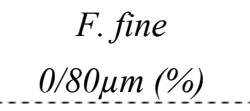 & 92.0 & 99.0 & 83.0 & 87.0 & 75.0 & 76.0 & 77.0 & 80.0 \\
\hline$\rho_{s}\left(\mathrm{~g} / \mathrm{cm}^{3}\right)$ & 2.50 & 2.64 & 2.57 & 2.55 & 2.27 & 2.51 & 2.53 & 2.34 \\
\hline$W L(\%)$ & 100 & 36 & 70 & 94 & 57 & 130 & 119 & 132 \\
\hline$I P(\%)$ & 62 & 15 & 20 & 44 & 23 & 70 & 67 & 67 \\
\hline$V B s(g / 100 \mathrm{~g} \mathrm{sec})$ & 3.3. & 1.2 & 2.2. & 2.3 & 1.1 & 2.9 & 1.4 & 2.4 \\
\hline Classe GTR & $F 11$ & $F 11$ & $F 12$ & $F 11$ & $F 11$ & $F 12$ & $F 11$ & $F 12$ \\
\hline
\end{tabular}

\subsection{Traitement des sédiments aux liants hydrauliques}

Les essais d'aptitude permettent d'appréhender le comportement des sédiments vis-à-vis d'un traitement aux liants hydrauliques défini. La figure 1 présente les principaux essais de caractérisation physique et mécanique ainsi que les critères recommandés par le Guide technique de Traitements des Sols à la chaux et/ou aux liants hydrauliques (GTS) pour une utilisation de matériaux en couche de forme (LCPC-SETRA, 2000).

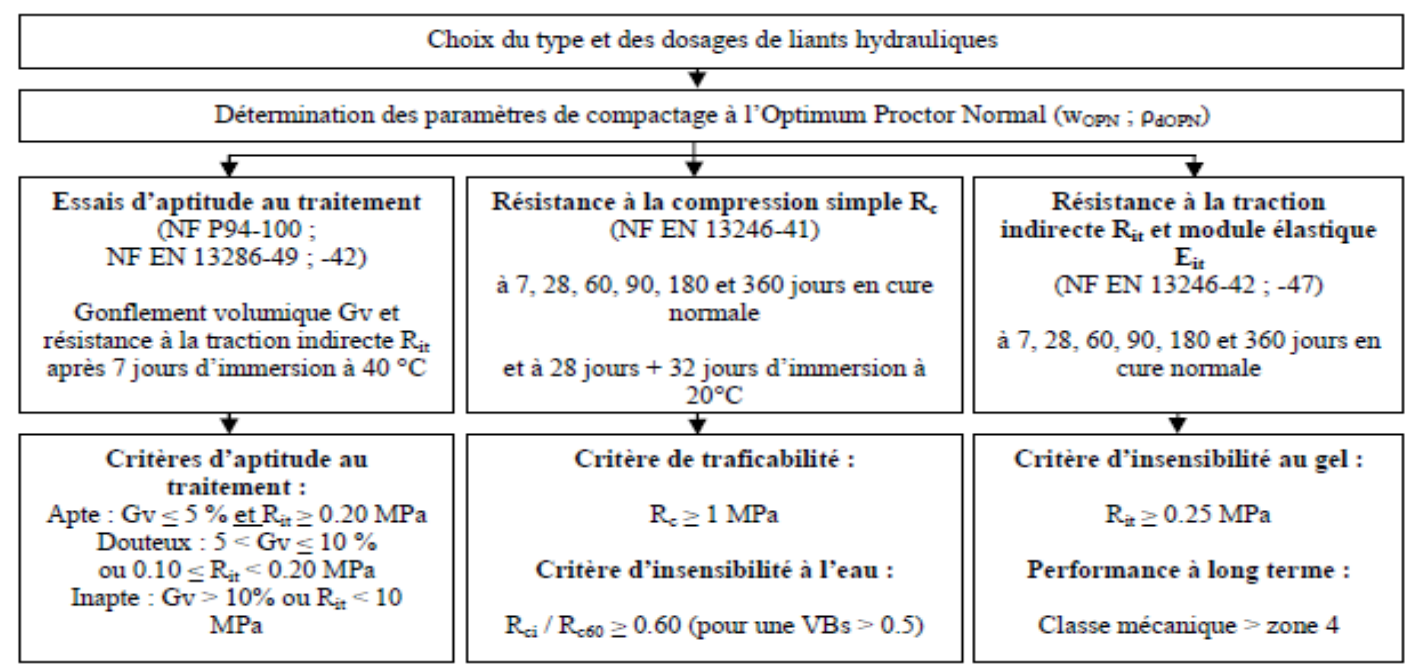

Figure 1. Progression d'une étude GTS pour une utilisation de matériaux traités en couche de forme (LCPC-SETRA, 2000). 


\section{a) Détermination des dosages en liants}

Le traitement adopté pour améliorer les caractéristiques de nos sédiments est un traitement mixte chaux vive - ciment CEMII 32.5. Pour la détermination du pourcentage de chaux vive, on se réfère à l'essai de point de fixation de la chaux. Cet essai consiste à mesurer le $\mathrm{pH}$ d'un mélange sédiment chaulé-eau (NF ISO 10390) et à déterminer pour quel dosage en chaux le $\mathrm{pH}$ de la suspension atteint une valeur de 12.4, favorable au développement des hydrates cimentaires. La figure 2 montre qu'à partir d'un pourcentage de chaux de $3 \%$, cette valeur de $\mathrm{pH}$ est atteinte. Par ailleurs, le GTS recommande, pour le traitement des sols de classes A1 et A2, l'utilisation d'un pourcentage de ciment CEMII 32.5 de 7\% (LCPC-SETRA, 2000). Dans le cadre de cette étude, pour répondre à des besoins économiques, nous avons utilisé un pourcentage de $6 \%$.

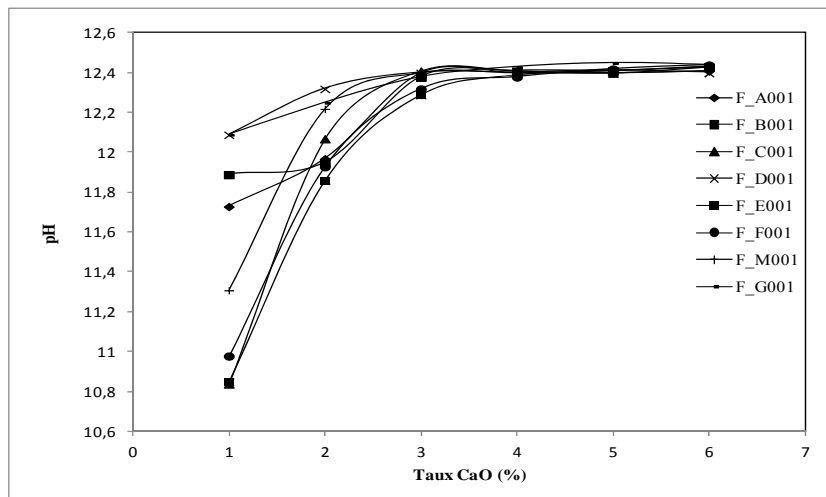

Figure 2. Evolution du pH en fonction du pourcentage de chaux.

\section{b) Détermination des paramètres de compactage}

Une fois la formulation de traitement fixée, il faut définir les paramètres de compactage de ces matériaux fins à l'énergie Proctor normal (selon la norme NF P94-093 actualisée par la norme NF EN 13286-2), soit la teneur en eau optimale wopN pour laquelle est obtenue la masse volumique sèche maximale $\rho_{\mathrm{dopN}}$. Les masses volumiques sèches $\rho_{\mathrm{dOPN}}$ des matériaux traités sont comprises entre 1.31 et $1.71 \mathrm{t} / \mathrm{m}^{3}$. Ces paramètres sont utiles lors de la confection des éprouvettes pour les essais d'aptitude.

\section{c) Confection des éprouvettes}

Pour les tests d'aptitude au traitement, pour chaque sédiment, 6 éprouvettes cylindriques de dimensions $5 \times 5 \mathrm{~cm}$ (diamètre $\times$ hauteur) ont été confectionnées, 3 pour la mesure du gonflement volumique $(\mathrm{Gv}), 3$ pour l'essai de résistance à la traction indirecte $\left(\mathrm{R}_{\mathrm{it}}\right)$. Elles ont été confectionnées par compression statique selon la norme NF EN 13286-53, à la teneur en eau optimale $w_{\text {OPN }}$ et à $96 \%$ de la masse volumique sèche optimale $\rho_{\text {dopN }}$. Après confection, les éprouvettes sont conservées pendant au moins $4 \mathrm{~h}$ à une humidité 


\section{XII ${ }^{\text {èmes }}$ Journées Nationales Génie Côtier - Génie Civil \\ Cherbourg, 12-14 juin 2012}

relative supérieure à $90 \%$ et à une température de $20{ }^{\circ} \mathrm{C}$, avant d'être immergées dans un bain thermostatique à $40^{\circ} \mathrm{C}$ pendant 7 jours.

Les éprouvettes cylindriques destinées aux mesures de résistance à la compression simple $\left(\mathrm{R}_{\mathrm{c}}\right)$ en cure normale, de dimensions $5 \times 10 \mathrm{~cm}$, sont compactées à $\mathrm{w}_{\mathrm{OPN}}$ et à $98,5 \%$ de $\rho_{\text {dopN }}$. Les éprouvettes destinées aux mesures de $\mathrm{R}_{\text {it }}$ en cure normale ont les même caractéristiques de compactage que les éprouvettes des tests d'aptitude. Les éprouvettes sont conservées dans des moules hermétiques, sans échange avec l'extérieur, à $20^{\circ} \mathrm{C}$.

\section{Résultats et discussion}

3.1 Aptitude des sédiments de dragage marins au traitement

Les résultats de gonflement volumique (Gv) sont repris dans le tableau 2. Pour les sédiments A001, E001, F001 et M001, le Gv n'a pas été mesuré car les éprouvettes immergées se sont effritées.

Tableau 2. Gonflements volumiques des sédiments traités.

\begin{tabular}{llll}
\hline Sédiments & Mesure Gv (\%) & Jugement & $C_{\text {MOC }}(\%)$ \\
\hline A001 & Non Mesurable (N.M.) & Inapte & 8 \\
B001 & 1 & Apte & 4 \\
$C 001$ & 6 & Douteux & 15 \\
$D 001$ & 5 & Douteux & 7 \\
E001 & N.M. & Inapte & 11 \\
F001 & N.M. & Inapte & 11 \\
G001 & 1 & Apte & 5 \\
M001 & N.M. & Inapte & 15 \\
\hline
\end{tabular}

L'observation de l'eau dans laquelle les sédiments ont été conservés pendant une semaine révèle une coloration jaunâtre et la présence de matières en suspensions. Ceci peut être expliqué, en partie, par la présence de matières organiques. En effet, les acides humiques et fulviques sont solubles en milieu basique (REKIK, 2007 ; DAVRANCHE, 2008). Cela pourrait expliquer la déstructuration des éprouvettes de sédiments pour lesquels les teneurs en M.O. sont proches de $10 \%$.

La figure 3 montre que les valeurs de $\mathrm{R}_{\mathrm{it}}$ après immersion restent largement inférieures à $0.10 \mathrm{MPa}$ : les 8 sédiments étudiés sont inaptes au traitement. Nous distinguons deux groupes de sédiments : ceux du groupe I sont ceux pour lesquels les teneurs en M.O. dépassent $10 \%$, ceux du groupe II ont les teneurs en M.O. les plus faibles. 


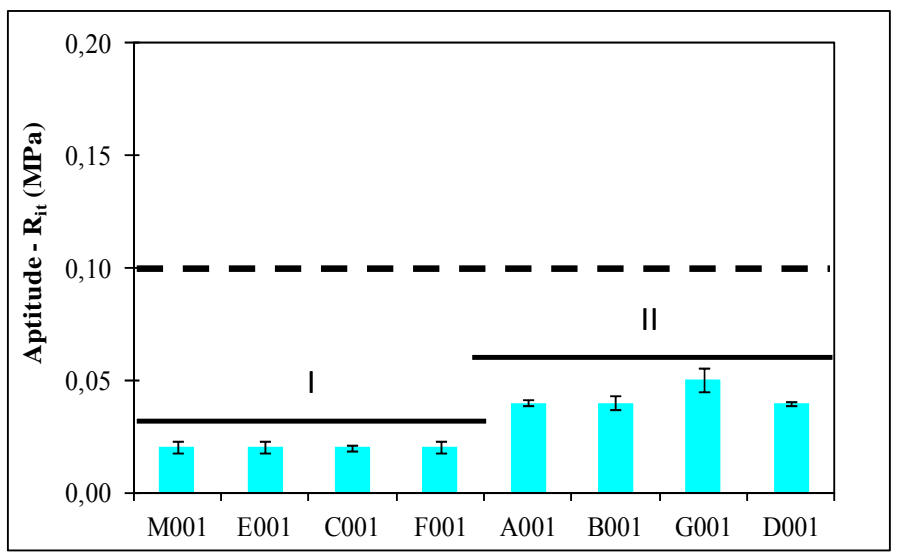

Figure 3. Résistances à la traction indirecte en aptitude des sédiments traités.

\subsection{Evolution des performances mécaniques}

La figure 4 indique l'évolution des $R_{i t}$ et des $R_{c}$ en cure normale. Après 28 jours de cure, tous les sédiments traités ont des résistances à la compression simple inférieures à $1 \mathrm{MPa}$, valeur cible pour garantir la traficabilité de la sous-couche routière après sa mise en œuvre.
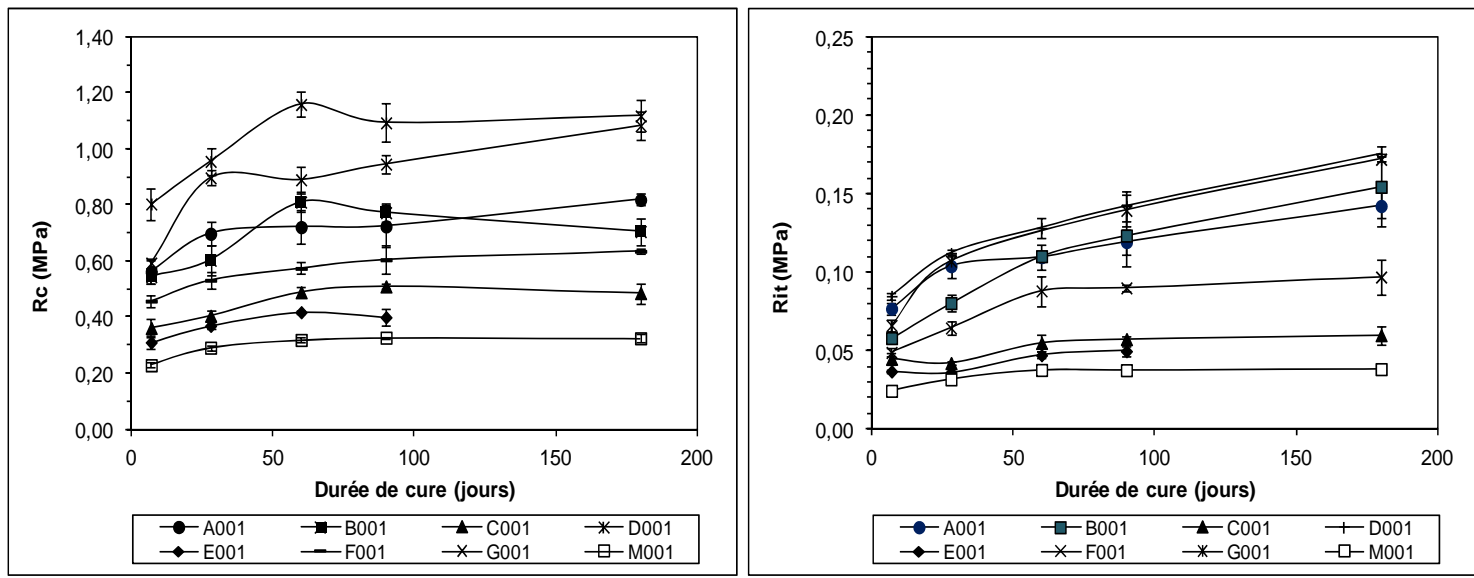

Figure 4. Résistances à la compression simple et à la traction indirecte des sédiments traités.

Les résistances à la traction indirecte des sédiments traités sont inférieures à $0.25 \mathrm{MPa}$, quelle que soit l'échéance, ce qui ne satisfait pas le critère de résistance au gel.

Nous remarquons un contraste entre les résistances développées par les différents sédiments. L'explication peut une nouvelle fois être rapprochée de la présence de M.O. En effet, les substances humiques interfèrent fortement dans les processus d'hydratation des liants. Ces interférences font considérablement chuter les résistances des matériaux traités à court et à moyen termes (KUJALA et MAKIKYRO, 1996 dans REY et al., 2000 ; TREMBLAY et al., 2002). De plus, s'ajoute probablement l'effet d'autres 


\section{XII ${ }^{\text {èmes }}$ Journées Nationales Génie Côtier - Génie Civil \\ Cherbourg, 12-14 juin 2012}

éléments chimiques présents dans les sédiments de dragage marins (chlorures, sulfates, éléments traces, etc.) qui peuvent retarder voire inhiber la formation d'hydrates nécessaires pour la montée en résistance des matériaux traités (LCPC-SETRA, 2000). Ainsi, il est à nouveau possible de distinguer le groupe I, constitué par les sédiments C001, E001, F001 et M001, avec les résistances les plus faibles, stables à partir de 60 jours, du groupe II, constitué par les sédiments A001, B001, D001 et G001, présentant les meilleures résistances et un développement des performances non stabilisé à 180 jours.

\subsection{Insensibilité à l'eau des sédiments traités}

Le tableau 3 présente les résultats de $R_{c}$ à 60 jours en cure normale, de $R_{c i}$ après 28 jours de cure normale suivi de 32 jours en immersion à $20^{\circ} \mathrm{C}$, ainsi que le rapport entre ces deux résistances. Les résultats sur le sédiment G001 n'ont pas été validés à ce jour. $\mathrm{Au}$ vu des résultats obtenus, quel que soit le sédiment considéré, les valeurs du ratio $\mathrm{R}_{\mathrm{ci}} / \mathrm{R}_{\mathrm{c} 60}$, lorsqu'elles sont supérieures au seuil de 0.60 , restent toujours proches de ce seuil, ce qui traduit, selon le GTS (LCPC-SETRA, 2000), une faible résistance à l'immersion. Les sédiments E001 et F001 présentent un ratio inférieur à 0.60 ce qui indique leur sensibilité importante à l'immersion.

Tableau 3. Insensibilisation à l'eau des sédiments traités.

\begin{tabular}{lllllllll}
\hline Sédiments & $M 001$ & $E 001$ & $C 001$ & $F 001$ & $A 001$ & $B 001$ & $G 001$ & $D 001$ \\
\hline$R_{c 60}(M P a)$ & 0.32 & 0.42 & 0.49 & 0.57 & 0.72 & 0.81 & 0.89 & 1.16 \\
$R_{c i}(M P a)$ & 0.23 & 0.23 & 0.29 & 0.30 & 0.47 & 0.60 & - & 0.76 \\
$R_{c i} / R_{c 60}$ & 0.71 & 0.55 & 0.60 & 0.52 & 0.65 & 0.74 & - & 0.66 \\
\hline
\end{tabular}

\section{Conclusion}

Cette étude, qui constituait la première phase du projet SETARMS, avait pour but de mesurer le potentiel qu'offre le traitement aux liants hydrauliques de sédiments de dragage portuaires dans l'optique d'une application en travaux routiers. Un traitement mixte à base de $3 \%$ de chaux vive et $6 \%$ de ciment CEM II $32,5 \mathrm{R}$ a permis de montrer que les résistances développées ne sont pas satisfaisantes pour une utilisation des sédiments en couche de forme. Cependant, les sédiments du groupe II ont montré une évolution des performances mécaniques au cours du temps, non stabilisée à 180 jours. Ce phénomène doit être exploité à l'avenir. Ainsi, l'étude de formulation sera poursuivie en intégrant par exemple :

- un prétraitement permettant de diminuer la teneur en M.O., par destruction chimique ou par dilution, avec l'apport d'un correcteur granulaire par exemple ;

- le potentiel pouzzolanique des sédiments D001, G001, A001 et B001 qui peut être mesuré indirectement en quantifiant les silicates de calcium hydratés (CSH) formés (INDRARATNA et al., 1991 ; IDIR, 2009) ; 


\section{Remerciements}

Les auteurs remercient l'ensemble des partenaires du projet SETARMS, en particulier l'Ecole des Mines de Douai pour sa participation à la caractérisation du sédiment M001, les sociétés Lhoist et Calcia, ainsi que l'Europe et le Conseil Régional de BasseNormandie, organismes financeurs.

\section{Références bibliographiques}

BOUTOUIL M. (2010). Présentation du projet SETARMS. II ${ }^{\text {nd }}$ International Symposium on Sédiment Management, I2SM, Casablanca, 11-13 may 2010.

DAVRANCHE M. (2008). Impacts des processus aux interfaces sol-eau sur la spéciation des éléments. HDR, Université de Limoges, $121 \mathrm{p}$.

IDIR R. (2009). Mécanismes d'action des fines et des granulats de verre sur la réaction alkali-silice et la réaction pouzzolanique. Thèse de doctorat, université de Toulouse, $292 \mathrm{p}$.

INDRARATNA B., NUTALAYA P., KOO K. S., KUGANENTHIRA N. (1991). Engineering behavior of a low carbon pozzolanic fly ash and its potential as a construction fill. Revue Canadienne de Géotechnique, Vol. 28. $\mathrm{n}^{\circ}$ 4, pp 542-555.

KUJALA K., MAKIKYRO M. (1996). Effect of humus on the binding reaction in stabilized soils. Grouting and Deep mixing, Ed. Yonekura Terashi et Shibazaki, Rotterdam, pp 415-420

LCPC-SETRA (2000). Réalisation des remblais et des couches de forme. Guide technique des terrassements routiers (GTR).

LCPC-SETRA (2000). Traitement des sols à la chaux et/ou aux liants hydrauliques Application à la réalisation des remblais et des couches de forme - Guide technique (GTS). $240 \mathrm{p}$.

REKIK B. (2007). Propriétés géotechniques des sédiments de dragage traités au ciment. Thèse de doctorat, Université de Caen, $122 \mathrm{p}$.

REY F., LEVACHER D., QUENEC'H J.-L. (2000). La valorisation par solidification/stabilisation à base de chaux et additifs de vases de dragage : résultats et perspectives. $\mathrm{VI}^{\text {èmes }}$ Journées Nationales Génie Civil-Génie côtier, 17-19 mai 2000, Caen, pp 377-386. doi:10.5150/jngcgc.2000.032-R

TREMBLAY H., DUCHESNE J., LOCAT J. and LEROUEIL S. (2002). Influence of the nature of organic compounds on fine soil stabilization with cement. Revue Canadienne de Géotechnique, Vol. 39, pp 535-546. 